\title{
Assessing Quality of Life Related to Oral Health and Glycemic Control in Type 2 Diabetic Patients - A Pilot Study Using GOHAl Test
}

\author{
Andreea Wagner ${ }^{1}{ }^{*}$, Elena Preoteasa ${ }^{1}$, Cristina Teodora Preoteasa ${ }^{2}$ \\ ${ }^{1}$ Department of Total Prosthesis, Carol Davila University of Medicine and Pharmacy, Bucharest, Romania \\ ${ }^{2}$ Department of Ergonomy, Carol Davila University of Medicine and Pharmacy, Bucharest, Romania \\ Email address: \\ andreea_wagner@yahoo.com (A. Wagner),dr_elena_preoteasa@yahoo.com (E. Preoteasa), cristina_5031@yahoo.com (C. T. Preoteasa) \\ ${ }^{*}$ Corresponding author
}

To cite this article:

Andreea Wagner, Elena Preoteasa, Cristina Teodora Preoteasa. Assessing Quality of Life Related to Oral Health and Glycemic Control in Type 2 Diabetic Patients - A Pilot Study Using GOHAI Test. Clinical Medicine Research. Vol. 9, No. 1, 2020, pp. 6-10.

doi: $10.11648 /$ j.cmr.20200901.12

Received: January 12, 2020; Accepted: February 3, 2020; Published: February 13, 2020

\begin{abstract}
Quality of life is an important outcome to accomplish, representing the final goal for the management of all general diseases. Diabetes mellitus is a chronic general disease with a high prevalence all over the world, mainly in adults and elderly. Patients with diabetes type 2 seem to have a worst quality of life in comparison to healthy ones. The objective of this study was to assess the oral health-related quality of diabetic type 2 patients in relation to general health status, medication and demographic factors. Methods: One hundred and thirty-five patients, 68 type $2 \mathrm{DM}$ and 67 nondiabetic were interviewed. Health-related quality of life was evaluated using the GOHAI questionnaire and related to general health, clinical and sociodemographic variables. Statistical analyses were determined using SPSS 18, t-test, ANOVA test, Pearson's correlation coefficient for quantitative variables and chi-test for qualitative variables. P value $<0.05$ was considered significant. Results: Among the studied population, the mean age was 66 for diabetic patients and 69 for the nondiabetic ones. Females, from rural environment, obese, with more than $7 \% \mathrm{HbA1C}$ levels reported worse quality of life. Smoking din not influenced significantly the results. Type 2 diabetic patients, with insulin therapy, with more than 10 years duration of disease, with other comorbidity present had lower results in all the three quality of life domains. Conclusion: We found that the quality of life in diabetic type 2 patients was lower in all the domains (psychological, physical, pain) in comparison with nondiabetic persons, but with little difference between study groups.
\end{abstract}

Keywords: Diabetes Mellitus, Health-related Quality of Life, GOHAI, General Health Status

\section{Introduction}

Diabetes is a chronic pancreatic disease which affects many people worldwide. The World Health Organization (WHO) estimated that the number of diabetic patients will increase to 439 millions till 2030. The most common form is type 2 diabetes which appears in $90 \%$ of individuals with diabetes, summing $10 \%$ of population over 65 years old worldwide [1].

The disease is also known as the fourth leading cause of death according to WHO [2]. In Romania diabetes is the 7th cause of death with 1.785300 diabetic patients $(12,4 \%$ of population) $[3,4]$.

Diabetes mellitus or type 2 diabetes is associated with hyperglycemia and can lead to microvascular complications (nephropathy, neuropathy and retinopathy) and accelerate the macro vascular complications (cardiovascular disease, diabetic foot). Oral changes involve xerostomia and impairment of healing process which lead to plaque accumulation, candidiasis, periodontitis, periodontal abscess, burning mouth syndrome.

People with chronic impairment, such as diabetes type 2 have to deal with a lot of problems which may affect their health related quality of life (HRQoL). Quality of life issues are important because they predict the capacity of self-management of the disease and long term health and well-being. 
Several studies in the literature demonstrated a negative impact of diabetes in health related quality of life of the patients, especially in the presence of complications [5-9].

The relationship between sociodemographic characteristics, Body Mass Index (BMI), duration of disease, type of medication, HbAlC levels, comorbidity and health related quality of life in type 2 diabetic patients is not so common in the literature. The main goal of this study was to assess the relation between these factors and health related quality of life in type 2 diabetic patients using GOHAI (Geriatric Oral Health Assessment Index) test. Questionnaires are reported as useful tools in evaluating health related quality of life of patients.

\section{Method}

We performed a cross-sectional study on a convenience sample of diabetic and non-diabetic patients. Diabetic patients were recruited from Nicolae Paulescu Hospital, Department of Diabetes, Bucharest. The control study group included nondiabetic patients receiving removable prosthodontic treatment in Department of Removable Prosthodontics, Carol Davila University of Medicine and Pharmacy, Bucharest. The study took place between January-December 2018.

We included patients over 40 years old, both sexes, which signed the informed consent prior to participation into the study. Patients were divided in two groups: A group with type 2 diabetes, with 2 subdivisions: a1-controlled diabetes- $\mathrm{HbA} 1 \mathrm{C}<7 \%$ and $\mathrm{a} 2$ uncontrolled diabetes- $\mathrm{HbA} 1 \mathrm{C}>=7 \%$ and $\mathrm{B}$ group-which included nondiabetic subjects. The protocol for the study was approved by the Ethics Committee for Health in Bucharest.

One questionnaire was designed to collect demographic and clinical data (gender, age, level of education, BMI, prosthetic status, smoking habits, comorbidity, $\mathrm{HbA1C}$ level, duration of diabetes, type of diabetic medication, microvascular and macro vascular complications in diabetic patients). The selected patients were administrated with GOHAI-Per 12 questionnaire and the responses were recorded by direct interview. Finally, patients were clinically intraoral examined by one single dentist.

Body mass index (BMI) was calculated from self-reported height and weight. It is the expression of weight (kilograms) divided by square of height (meters). According to WHO [4] the values are normal: $18-24.9 \mathrm{~kg} / \mathrm{m}^{2}$, overweight: $25-29.9$ $\mathrm{kg} / \mathrm{m}^{2}$ and obesity $>=30 \mathrm{~kg} / \mathrm{m}^{2}$. Duration of disease was categorized as: "less than 5 years", "5-10 years", "more than 10 years".

The glycemic control is one of the major objectives in clinical management of diabetic patients. The level of $\mathrm{HbA1C}$ was recorded from patient observation sheet of the hospital. The results of glycemic control in diabetic patients were categorized as: normal: $\mathrm{HbA} 1 \mathrm{C}<=6.9 \%$, moderate: $\mathrm{HbA} 1 \mathrm{C}$ $7-8.9 \%$, poor control: $\mathrm{HbA} 1 \mathrm{C}>=9.0 \%$. In control group study patients, glycaemia was determined directly, using \& jeun technique and a well-known glucometer device (ACCU CECK-Roch Diagnostics). All patients in B group were having less than $126 \mathrm{mg} / \mathrm{dl}$ blood sugar levels.
As for the complications we recorded the number and type of microvascular and macro vascular ones, in group A.

Treatment regimens for type 2 diabetes were classified as: "diet and physical activity", "oral hypoglycemic agents", "insulin therapy", "both hypoglycemic agents and insulin".

As for general health, every other comorbidity present were recorded.

The quality of life was assessed based on GOHAI questionnaire-interview, which consists of 12 items. The reproduction and reliability of GOHAI-12 has been confirmed by others literature studies. In this study the Romanian version of GOHAI, previously validated, was used [10]. This test covers 12 questions with 5 possible answers, scored as following: Never $=1$; Seldom $=2$; Sometimes $=3$; Often=4; Always $=5$.

The information is related to oral problems which appeared in the past 3 months, in three dimension of oral health quality of life: physical function, pain and discomfort, psychological function. Total of GOHAI scores was termed: ADD-GOHAI (Additive score of GOHAI) it ranges between 12 minimum 60 maximum. Higher score indicates higher oral health related quality of life. The levels of quality of life are scored as good (more than 40), average (between 30-40) and poor (less than 30).

To avoid possible misunderstanding in scoring the answers, we divided the possible answers in two subgroups in which the score for the answers: "Sometimes", "Seldom", "Never" would be 1 for each questions and for the answers "Always", "Often" would be 0. SC-GOHAI (Simple count) score in this method would range from 0 to 12 ; a higher score would indicate a higher quality of life.

Statistical analyses were conducted using SPSS 18 software and we used: t-test, ANOVA test, Pearson's correlation coefficient for quantitative variables and chi-test for qualitative variables. $\mathrm{P}$ value $<0.05$ was considered significant.

\section{Results}

\subsection{Socio-demographic Variables and Medical History}

As per the study criteria, the 135 patients were included in the study sample, from which $68(50,4 \%)$ with Diabetes mellitus, and $67(49,6)$ nondiabetic patients. From the diabetic patients, 70,6\% $(\mathrm{n}=48)$ had uncontrolled diabetes mellitus, and $29,4 \%(n=20)$ had controlled diabetes.

The glycemic control in diabetic patients was found to be poor $(\mathrm{HbA} 1 \mathrm{C}>7 \%)$ in a high percent of people $(44,1 \%)$. The mean of sugar blood level in nondiabetic patients was 82,4 , with higher value in people aged over 70 years old, in both sexes ( $85.2 \%$ females and $83.6 \%$ males).

We observed that out of 68 diabetic patients, $8(11,7 \%)$ had a history of diabetes less than 5 years, $42(61,76 \%)$ more than 10 years and $18(26,4 \%) 5-10$ years.

The number of obese diabetic patients was higher in females, over 10 years old of disease $(40,5 \%)$.

There were diabetic patients being treated with AO $(30.88 \%)$, insulin monotherapy $(32,35 \%), 35.29 \%$ were taking 
a combination of $\mathrm{AO}$ and insulin and only $1.4 \%$ were on regular diet without medical treatment.

Patients were prescribed between 1-4 medication for other general treatment. HBP was the most frequent comorbidity $(76.5 \%)$ and retinopathy the most frequent complication of Diabetes mellitus observed (47.1\%) as seen in "Table 1".

Table 1. Medical history of diabetes mellitus (DM) patients.

\begin{tabular}{lll}
\hline Variable & number & \% \\
\hline HbA1C level & & \\
Normal & 20 & 29.4 \\
Moderate & 30 & 44.1 \\
Poor & 18 & 26.5 \\
Medication type for DM & & \\
Diet and exercises & 1 & $1.47 \%$ \\
Insulin & 22 & $32.35 \%$ \\
ADO & 21 & $30.88 \%$ \\
ADO and insulin & 24 & $35.29 \%$ \\
Duration of disease & & \\
$<5$ years & 8 & $11.8 \%$ \\
$5-10$ years & 18 & $26.5 \%$ \\
$>10$ years & 42 & $61.8 \%$ \\
\hline
\end{tabular}

\begin{tabular}{lll}
\hline Variable & number & $\%$ \\
\hline Comorbidity and complications of DM & & \\
Retinopathy & 32 & $47.1 \%$ \\
Polyneuropathy & 35 & $51.5 \%$ \\
Nephropathy & 9 & $13.2 \%$ \\
Periodontitis & 20 & $29.4 \%$ \\
HBP & 52 & $76.5 \%$ \\
CV disease & 11 & $16.2 \%$ \\
Obesity & 11 & $16.2 \%$ \\
Number of medications for other & & \\
chronic diseases & & \\
0 & 4 & $5.9 \%$ \\
1 & 7 & $10.3 \%$ \\
2 & 19 & $27.9 \%$ \\
3 & 13 & $19.1 \%$ \\
4 & 15 & $22.1 \%$ \\
\hline
\end{tabular}

As per socio-demographic variables included, we observed that the groups studied were quite similar, as seen in "Table 2 ". However, we found a higher proportion of obese people through diabetic patients $(33,8 \%)$, with a higher range in uncontrolled ones $(37.5 \%)$

Table 2. Patients' characteristics.

\begin{tabular}{|c|c|c|c|}
\hline Characteristics & & Group A (DM patients) $n=68$ & Group B (nondiabetic patients) $n=67$ \\
\hline \multirow[t]{2}{*}{ Sex } & Male (no;\%) & $\mathrm{n}=23(33.8 \%)$ & $\mathrm{n}=26(38.8 \%)$ \\
\hline & Female (no;\%) & $\mathrm{n}=45(66.6 \%)$ & $\mathrm{n}=41(61.2 \%)$ \\
\hline \multicolumn{2}{|l|}{ Age (mean) } & 66 years old & 69 years old \\
\hline \multirow[t]{2}{*}{ Region } & Urban (no;\%) & $41(60.3 \%)$ & $47(70.1 \%)$ \\
\hline & Rural (no;\%) & $27(39.7 \%)$ & $20(29.9 \%)$ \\
\hline \multirow[t]{2}{*}{ BMI } & Normal (no;\%) & $20(29.4 \%)$ & $27(40.3 \%)$ \\
\hline & Obese (no;\%) & $23(33.8 \%)$ & $4(6.0 \%)$ \\
\hline \multirow[t]{3}{*}{ Smoking } & Yes & $30(44.1 \%)$ & $29(43.3 \%)$ \\
\hline & No & $38(55.9 \%)$ & $38(56.7 \%)$ \\
\hline & No formal ducation & $2(2.9 \%)$ & $4(6.0 \%)$ \\
\hline \multirow{2}{*}{ Education Level } & Primary & $17(25.0 \%)$ & $14(20.9 \%)$ \\
\hline & Secondary & $37(54.4 \%)$ & $37(55.2 \%)$ \\
\hline
\end{tabular}

The majority of patients ware from urban region $(60,3 \%$ in group $\mathrm{A}$ and $70,1 \%$ in group B) and most of them were females $(66,2 \%$ in diabetic patients and $61,2 \%$ in nondiabetic subjects.)

Among 135 patients, the history of cigarette smoking was present in $43,7 \%$ cases and was higher in diabetic male patients $(56.7 \%)$ aged between $60-69$ years old (50\%).

Mean BMI for the responders was $22.03 \%$ for healthy ones and $28,35 \%$ for DM patients. In both groups we found a medium level of education (high school), no matter the gender, as following: $54,8 \%$ in urban patients and $53,8 \%$ in patients living in rural zones.

Responses to GOHAI items tended to be "never",

"sometimes" and "seldom".

The mean ADD-GOHAI score was 27.14, which is assessed as poor (less than 30). The quality of life of diabetic patients was poor in all the domains (psychological, physical, pain) in comparison with nondiabetic persons, as shown in "Figure 1".

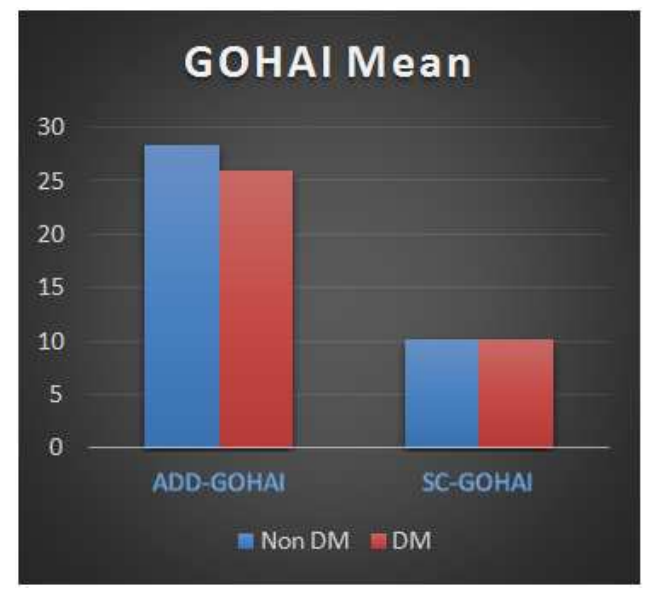

Figure 1. Mean of GOHAI score in nondiabetic and diabetic mellitus patients.

The quality of life in comparison to sex showed that no significant differences in the quality of life scores between females and males, and it was rated as average $(27.84 \%$ vs 
$25.87 \%)$.

Smoker diabetic patients have poorer Quality of life in comparison with non-diabetic patients as seen in "Figure 2".

\section{Smoking and GOHAI Mean}

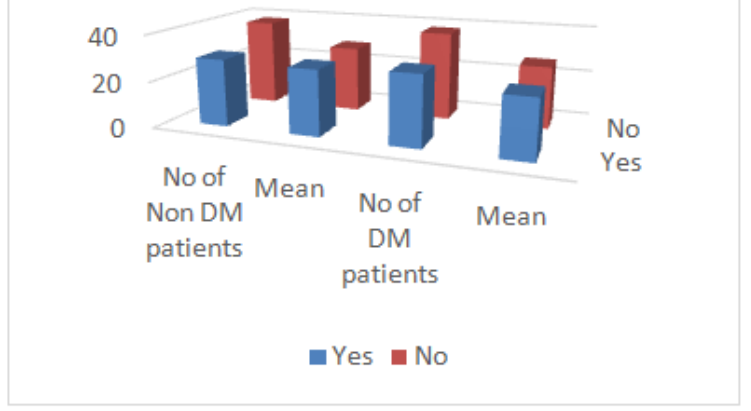

Figure 2. Quality of life and smoking habits.

\subsection{Quality of Life, Health Status, Relationship with HbA1C}

Medication has not a big influence in results of quality of life in both studied groups. In our study, patients with more than $8 \% \mathrm{HbA} 1 \mathrm{C}$ level had a lower quality of life score in contrast to the others, as shown in "Figure 3".

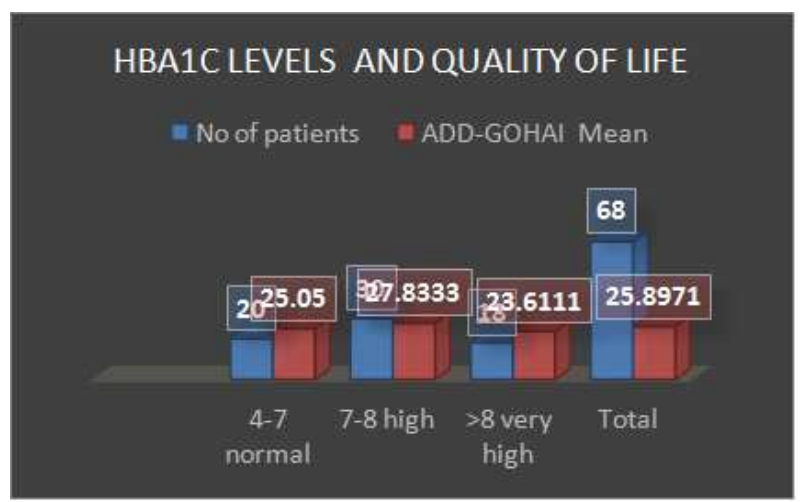

Figure 3. Level of HbAlC and Quality of Life in DM type 2 patients.

\section{Discussion}

The objective of this study was to assess the quality of life of type 2 diabetic patients in relation to general health, clinical and demographic characteristics.

We found that patients with type 2 diabetes had a negative impact on Quality of life, with low scores in GOHAI test (25.8). This is supported by other studies [11-14], which concluded that diabetes has an adverse effect in quality of life of the patients. The majority of the subjects studied were females (64.4\%), although in other studies the male proportion was higher $[11,18]$. The mean age was found to be 66 years as in other study [11] in which the mean age reported was 60 years old. The majority of patients were in group 60-69 years old, as observed in other literature studies $[15,16]$. One study [17] observed that in developing countries the majority of diabetic patients had between 45-65 years old. We observed that age is an important characteristic in quality of life. As the age increase, the lower level of quality of life scores appears $(p=0.632)$. In the proportion of more than 70 years old patients we observed a strong correlation to lower level of quality of life levels $(\mathrm{p}=0.353)$

We observed that even the number of urban diabetic patients was higher (89), the level of ADD -GOHAI score in rural patients was lower. This can be explained by less accessibility and interest in treatment of diabetes in rural patients. In our study, majority of diabetic patients had a history more than 10 years $(61.8 \%)$ and their quality of life score was poor in comparison to those with a history of less than 10 years, similar to other literature studies [14, 17]. We observed that as for the duration of history of the disease increased there was a significant decrease in the Quality of life, the same as in other published study [11].

Smoking was associated with lower levels of quality of life $(\mathrm{ADD}-\mathrm{GOHAI}=26,5)$ but without strong statistical significance $(p=6.14)$.

The level of education was also an important aspect and we found lower quality of life scores in non-educated type 2 diabetic patients (ADD-GOHAI $=30)$.

The patients with a BMI of less than $18,4 \mathrm{Kg} / \mathrm{m}^{2}$ had a better quality of life than patients with a BMI $>25 \mathrm{Kg} / \mathrm{m}^{2}$. This conclusion is reported in many other studies conducted on diabetic patients $[19,20]$.

Patients with $\mathrm{HbA} 1 \mathrm{C}$ levels $7-8 \%$ had a better quality of life in comparison to other subgroups. This fact was described also in other study [21] in which they observed that poor quality of life was associated with more that $8 \% \mathrm{HbA} 1 \mathrm{C}$ levels.

We found poor oral health-related quality of life in both groups of patients, with little difference in diabetic and nondiabetic persons. This result may be due to the small dimension of the study. The proportion of comorbidity was higher in type 2 diabetic patients (35.3\%) and $25.4 \%$ in control, nondiabetic group. The number of gastric problems in diabetic type 2 patients was higher (19.1\%) while the control group had much more comorbidities such as HBP (47.8\%), gastric (14.9\%), psychological (53,7\%), kidney disease (3\%). Type 2 diabetic patients on insulin therapy had a worse quality of life than patients with $\mathrm{HO}$ treatment.

Also type 2 diabetic patients were having lower quality of life levels in all the GOHAI questionnaire domains in comparison to nondiabetic patients $(25,8$ vs 28 in nondiabetic study population), similar to other literature studies [9, 22].

Some limitations should be considered when interpreting the results of the present study. A larger sample is needed for the generalization of our findings. Our population was collected in a central public hospital and might not be representative for diabetic population in other care centers or in primary care. Also, more of the patients interviewed were of low sociocultural level which may have limited their understanding and answering questions of GOHAI test. Further investigations are indicated to adjust the dimensions of GOHAI scores, demographic and clinical variables considered. Using qualitative tests could be useful to obtain complementary data to others quantitative studies, allowing more accurate validation of some results. 


\section{Conclusions}

Diabetes mellitus continues to be one of the major contemporary health-related issue. In addressing the epidemic illness, a primary therapeutic goal is quality of life. Diabetes affects all major domain of quality of life evaluated with GOHAI questionnaire (physical, psychological, pain and discomfort). We found that being female, rural, non-educated, obese, type 2 diabetic with $\mathrm{HbA} 1 \mathrm{C}$ level $>8 \%$, with insulin treatment and with more than 10 years of the disease are some characteristic associated with worse age-adjusted oral health-related quality of life in diabetic type 2 patients vs nondiabetic population. The presence of comorbidity and complications has an adverse effect on quality of life, but with no strong statistical significance. In future studies it would be advisable to implement this questionnaire in a larger sample of population.

In conclusion, Type 2 diabetes have a negative impact in oral health-related quality of life. It is recommended for this patients to have a strict glycemic control, enabling to prevent complication and disease progression. Knowing the risk factors, national strategy programs should be implemented to overcome this epidemic disease.

\section{Competing Interests}

The authors declare that they have no competing interests.

\section{References}

[1] Shaw JE Sicree R A and Zimmet PZ. Global estimates of prevalence of diabetes for 2010 ans 2030, Diabetes Res Clin Pract. 2010; 87 (1) 4-14.

[2] WHO2019. Country and regional data on diabetes [Internet]. Available from: https://www.who.int/diabetes/facts/world_figures/index5.html

[3] Prevalence of diabetes mellitus and prediabetes in the adult Romanian population: PREDATORR study. J Diabetes. 2016; 8 (3): $336-44$.

[4] http://insp.gov.ro/sites/cnepss/wpcontent/uploads/2018/11/AN ALIZA_Diabet_2018_rev.pdf

[5] Fal AM, Jankowska B, Uchmanowicz I, Sen M, Panaszek B, Polanski J. Type 2 diabetes quality of life patients treated with insulin and oral hypoglycemic medication. Acta Diabetol. 2011; 48: $237-242$.

[6] Wexler DJ, Grant RW, Wittenberg E, et al. Correlates of health-related quality of life in type 2 diabetes. Diabetologia. 2006; 49: 1489-1497.

[7] Lee WJ, Song KH, Noh JH, Choi YJ, Jo MW. Health-related quality of life using the EuroQol 5D questionnaire in Korean patients with type 2 diabetes. J Korean Med Sci. 2012; 27: 255-260.
[8] Andayani TM, Ibrahim MIM, Asdie AH. The association of diabetes-related factor and quality of life in type 2 diabetes mellitus. Int J Pharm Pharm Sci. 2010; 2: 139-145.

[9] Sepúlveda E, Poínhos R, Constante M, Pais-Ribeiro J, Freitas P, Carvalho D. Health-related quality of life in type 1 and type 2 diabetic patients in a Portuguese central public hospital. Diabetes Metab Syndr Obes. 2015; 8: 219-226.

[10] Murariu A, Hanganu C, Bobu L. Evaluation of the Reliability of the Geriatric Oral Health Assessment Index (GOHAI) in Institutionalised Elderly in Romania: A Pilot Study, OHDM. 2010; 9 (1): 64-68.

[11] Prajapati V, Raushan B, Acharya L, Seshadri S. Assessment of quality of life in type II diabetic patients using the modified diabetes quality of life (MDQoL)-17 questionnaire. Brazilian Journal of Pharmaceutical Sciences. 2017; 53 (4): 75-90.

[12] Somappa HK, Venkatesha M, Prasad R. Quality of life assessment among type 2 diabetic patients in rural tertiary centre. Int J Med Sci Public Health. 2014; 3: 415-417.

[13] Anumol $\mathrm{M}$ et al., American International Journal of Research in Humanities, Arts and Social Sciences. 2014; 7: 197-200.

[14] Akinci F, Yildirim A, Gözü H, Sargin H, Orbay E, Sargin M. Assessment of health related quality of life (HRQoL) of patients with type 2 diabetes in Turkey. Diabet Res Clin Pract. 2008; 79 (1): 117-23.

[15] Rubin R, Peyrot M. Quality of life and diabetes. Diabetes metabolism. 1999; 15: 205-2018.

[16] King H, Aubert RE, Herman WH. Global burden of diabetes, 1995-2025: prevalence, numerical estimates, and projections. Diabetes Care. 1998; 21 (9): 1414-31

[17] Othman W, Muttalib K, Bakri R, Doss J, Salleh N, Chen S. Validation of the Geriatric Oral Health Assessment Index (GOHAI) in the Malay Language. Journal of Public Health Dentistry. 2006; 66 (3): 199-204.

[18] Eljedi A, Mikolajczyk RT, Kraemer A, Laaser U. Health related quality of life in diabetic patients and controls without diabetes in refugee camps in the Gaza strip: a cross-sectional study. BMC Public Health. 2006; 6: 268-275.

[19] Trikkalinou A, Papazafiropoulou AK, Melidonis A. Type 2 diabetes and quality of life. World J Diabetes. 2017; 8 (4): 120 129.

[20] Hlatky MA, Chung SC, Escobedo J, Hillegass WB, Melsop K, Rogers W, et al. BARI 2D Study Group. The effect of obesity on quality of life in patients with diabetes and coronary artery disease. Am Heart J. 2010; 159 (2): 292-300.

[21] Stanetic K, Savic S, Racic M. The quality of life of patients with type II diabetes mellitus. Општа медицина. 2012; 18 (3/4): 70-7.

[22] Nikbin A, Bayani M, Jenabian N, Khafri S, Motallebnejad M. Oral health-related quality of life in diabetic patients: comparison of the Persian version of Geriatric Oral Health Assessment Index and Oral Health Impact Profile: A descriptive-analytic study. J. Diabetes Metab Disord. 2014; 13 (1): 13-32. 